\section{Finite Element Modeling of CRaCk Band Propagation}

\section{By Zdeněk P. Bažant, ${ }^{1}$ F. ASCE and Luigi Cedolin ${ }^{2}$}

Asstract: The propagation of blunt smeared crack bands through a finite element mesh in plain or reinforced concrete structures is studied. A previous work, which demonstrated (approximate) independence of results from the chosen mesh size, is extended by demonstrating the same for the changes in mesh orientation with regard to the crack band and to the steel bars. Bond slip
of bars must also be considered. This is satisfactorily accomplished by extendof bars must also be considered. This is satisfactorily accomplished by extending the previously proposed concept of free bond slip length for the cases of
skew meshes and skew bars. In the presence of reinforcement, the previous formula for the equivalent strength giving approximately the same results as the energy criterion must be expanded by a term which corresponds to the third term of the asymptotic expansion of displacements near the tip of a sharp crack. The energy criterion is shown to give consistent results for different types of fracture specimens. However, the equivalent strength calibrated for the center-cracked tensile specimen leads to a greater error (about $15 \%$ ) when applied to bent specimens. Thus, the similar equivalent strength approach is acceptable only if fine meshes are used although correct convergence appears to occur as the mesh size tends to zero.

\section{INTRODUCTION}

In finite element analysis, it is possible to represent cracking either as sharp and isolated interelement cracks or as bands of cracks imagined to be continuously distributed over the element. The latter method is more convenient for programming since it requires only a change in the matrix of incremental elastic constants of the material. For concrete, this method is also more realistic since the inhomogeneity of the material causes the cracking to be spread over a larger zone.

To decide whether a distributed (smeared) blunt crack band in a finite element mesh would extend, the normal procedure has been to compare the tensile stress in the element just ahead of the crack band to a specified tensile strength. Recently, it has been demonstrated, however, that this method is unobjective in that the results may strongly depend on the chosen element size $(1,2,4)$. An example of a reinforced concrete panel in which a four fold decrease in the element size causes the load

'Prof. and Dir., Center for Concrete and Geomaterials, Northwestern Univ., Technological Inst., Evanston, Ill. 60201.

${ }^{2}$ Visiting Scholar, Northwestern Univ., Evanston, Ill.; Prof. on Leave, School of Reinforced Concrete, Politecnico di Milano, Milano, Italy.

Note.-Discussion open until June 1, 1983. To extend the closing date one month, a written request must be filed with the ASCE Manager of Technical and Professional Publications. The manuscript for this paper was submitted for review and possible publication on September 21, 1981. This paper is part of the Journal of Structural Engineering, Vol. 109, No. 1, January, 1983. OASCE, ISSN 0733-9445/83/0001-0069/\$01.00. Proc. No. 17618. needed for further crack band extension to drop to one-half has been demonstrated. The problem was eliminated by introducing for the crack band extension an energy criterion $(1,2)$, which represents a refinement of Rice's criterion (5) for the extension of a notch. Further, it was found that, for reinforced concrete, objective results can be obtained only if the bond slip of reinforcing bars in the vicinity of the crack band is taken into account (2).

Although the aforementioned basic energy criterion $(1,2)$ is general, the previous numerical examinations were limited to the special case when the direction of cracks coincides with that of the mesh lines and the reinforcing bars are normal or parallel to the crack. In this paper, we extend the previous work by numerical studies of zig-zag skew crack bands in the finite element mesh and of reinforcement which is skew with regard to the mesh lines. Furthermore, since the previous numerical demonstrations were limited to a center-cracked rectangular panel we examine another typical situation, the case of the three-point bent specimen.

Further, we focus attention on the concept of equivalent strength, i.e. a tensile strength criterion in which the strength value is adjusted to give the correct energy release rate for any element size. This convenient method, which can be readily implemented in the existing finite element programs, has so far been numerically examined (1) only for the case of a center-cracked rectangular panel and for unreinforced concrete. This attractive approach is examined here for other specimen geometries, and we find that some refinements as well as a reassessment of applicability are found to be necessary. Finally, we indicate some results on the effectiveness of using various types of finite elements. Because of space limitation, the questions of applicability of the crack band model for fracture of concrete and the determination of material parameters and their variation are left to a separate study (3).

\section{Review of Caack Band Paopagation Caiterion}

Consider the two-dimensional situation sketched in Fig. 1 where the crack band of initial length $a$ advances by the length of one element, $\Delta a$. The energy release rate, $\mathscr{G}$, i.e., the energy released by the structure due to the cracking per unit length of the crack band, may be approximated as $\mathscr{G} \simeq-\Delta U / \Delta a$, in which $\Delta U$ is the energy released by the structure into the element that cracks. This approximation is second-order accurate if it is referred to the theoretical crack band length $a+\Delta a / 2$. As an extension of a previous formula by Rice (6), the energy release may be calculated as $\Delta U=\Delta W_{\Delta V}+\Delta L$ in which $(1,2)$

$\Delta W_{\Delta V}=-\int_{\Delta V} \frac{1}{2}\left(\sigma_{i j}^{c^{0}} \epsilon_{i j}^{0}-E_{c}^{\prime} \epsilon_{11}^{0^{2}}\right) d V \ldots \ldots \ldots \ldots \ldots \ldots \ldots \ldots \ldots \ldots \ldots \ldots \ldots \ldots \ldots \ldots$
$\Delta L=\int_{\Delta S} \frac{1}{2} \Delta T_{c i}^{0}\left(u_{i}-u_{i}^{0}\right) d S+\int_{\Delta V} \frac{1}{2} \Delta f_{c i}^{0}\left(u_{i}-u_{i}^{0}\right) d V \ldots \ldots \ldots \ldots \ldots \ldots$

The first expression represents the release of energy from the element of volume (or area) $\Delta V$ into which the cracks spread; $\sigma_{i j}^{0}$ and $\epsilon_{i j}^{0}$ represent the stresses in concrete and the strains in volume $\Delta V$ before the crack 


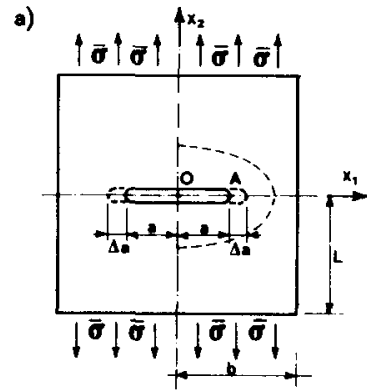

\section{b)} c)

\section{d)}
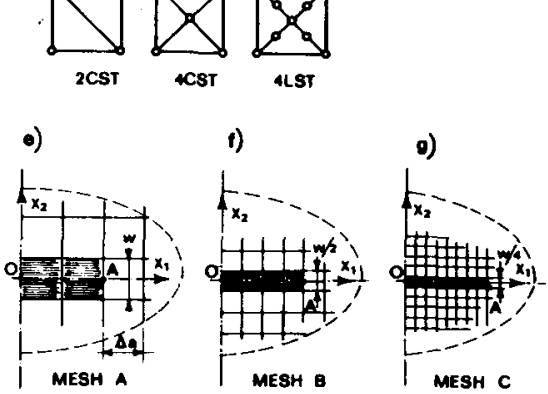

FIG. 1. (a) Center-Cracked Panel; (b-d) Types of Quadrliateral Finite Elements Examined; (0-g) Mesh Refinements Used in Examples

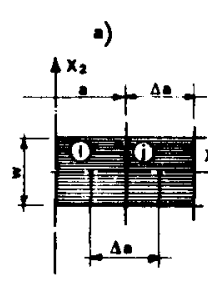

d)
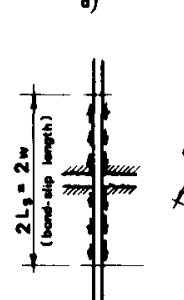

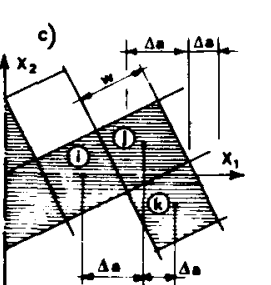

1)

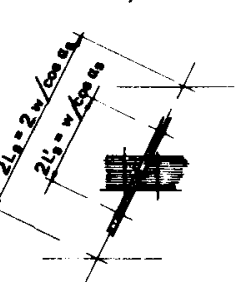

FIG. 2.-(a-c) Straight and Zig-Zag Crack Bands in Parallel and Inclined Finite Element Meshes; ( $\alpha-f)$ Bond-Sllp Length and Its Representation for Inclined Reinforcing Bars

extension; subscripts $i, j$ refer to cartesian coordinates $x_{i}(i=1,2) ; E_{c}^{\prime}=$ $E_{c}$ for plane stress and $E_{c}^{\prime}=E_{c} /\left(1-\nu_{c}^{2}\right)$ for plane strain, $E_{c}=$ Young's modulus for plain concrete and $v_{c}=$ Poisson ratio. The second term (Eq. 2) represents the work of the rest of the structure on the concrete contained in volume $\Delta V ; S$ denotes the boundary of the element of volume $\Delta V ; u_{i}^{0}, u_{i}=$ the displacements before and after the crack extension; $\Delta T_{c_{i}}^{0}=$ the surface tractions necessary to balance the initial stresses $\sigma_{i j}^{c^{0}}$ if the body would not deform during the crack extension; and $\Delta f_{c_{i}}^{0}=$ the volume forces representing the smeared bond forces between reinforcement and concrete needed to balance the initial stresses $\sigma_{i j}^{c^{0}}$. In numerical implementation, the integrations over $\Delta V$ and $\Delta S$ are approximated by sums over the nodal values.

The energy release rate must satisfy the condition $\mathscr{G} \leq \mathscr{G}_{c r}$ in which $\mathscr{G}_{c r}$ is a material property representing the critical value of the energy release rate; $\mathscr{G}=\mathscr{G}_{c r}$ indicates crack band propagation. The value of $\mathscr{G}_{c r}$ is not necessarily constant unless the structure is large. The value of $\xi_{c r}$ must be determined, however, by other considerations, and in the present analysis is assumed to be given.

For the purpose of analyzing fracture propagation of arbitrary and unknown direction, it is proper to require the mesh to be uniform near the crack band, since a nonuniformity of element size might favor certain crack directions over others. Therefore, we restrict attention to square meshes of finite elements.

\section{Zig-Zag Skew Chack Band and Skew Reinforcement}

In the numerical studies carried out so far $(1,2,4)$, the direction of crack propagation was assumed to be parallel to the lines of the finite element mesh (Fig. 2(a)). For general applications, we must allow, however, propagation of cracks in any direction, and it is one main attractive aspect of the blunt smeared crack band modeling that cracks of any direction can be easily simulated just by introducing orthotropic elastic moduli, with a proper direction of the orthotropy. The objectivity of the energy criterion (in the sense of independence of the analyst's choice of mesh) has so far been demonstrated only for the case of cracks parallel to the mesh lines. The condition of objectivity also requires, however, that the results for a given problem must be approximately the same when the finite element mesh is rotated with regard to the crack direction, while everything else remains the same.

In the rotated mesh, the crack band is skew and must have a zig-zag boundary. This is strictly a consequence of the modeling; the real boundary of the crack band should be imagined to be smooth. Therefore, the length of the crack advance, $\Delta a$, as considered in the energy criterion, cannot be considered as the length of the element along the mesh line $(w$ in Fig. 2(b and $c)$ ). Rather, we must measure $\Delta a$ in the direction of the crack band, and so we must consider a projection on axis $x_{1}$ (Fig. $2)$. Recalling that the approximation $\Delta U / \Delta a$ is second-order accurate when referred to the crack band length ending at the center of the element, we define $\Delta a$ for the zig-zag band as the projection of the distance between the centroids of two neighboring elements onto the crack direction $x_{1}$ (Fig. 2(b and $\left.c\right)$ ). (This is correct, of course, only if the crack direction is assumed to be such that the cracks are of Mode I type.)

We should keep in mind, however, that this is not the only reasonable possibility. We could, e.g., identify $\Delta a$ with the projection of the distance between the element corners farthest in the direction of propagation and $a$ with the center of this distance. However, the accuracy of this approach was not investigated since for programming the use of the centroid is much more convenient.

The parallel and rotated square meshes considered for the right half of the center-cracked panel, the same panel as that analyzed in Ref. 2, are shown in Fig. 3 (cases la and 1b). Fig. 3, case 1b, shows the zig-zag band. Also shown is the reinforcing grid and the free bond slip length for the bars crossing the crack band, which were determined in the same 

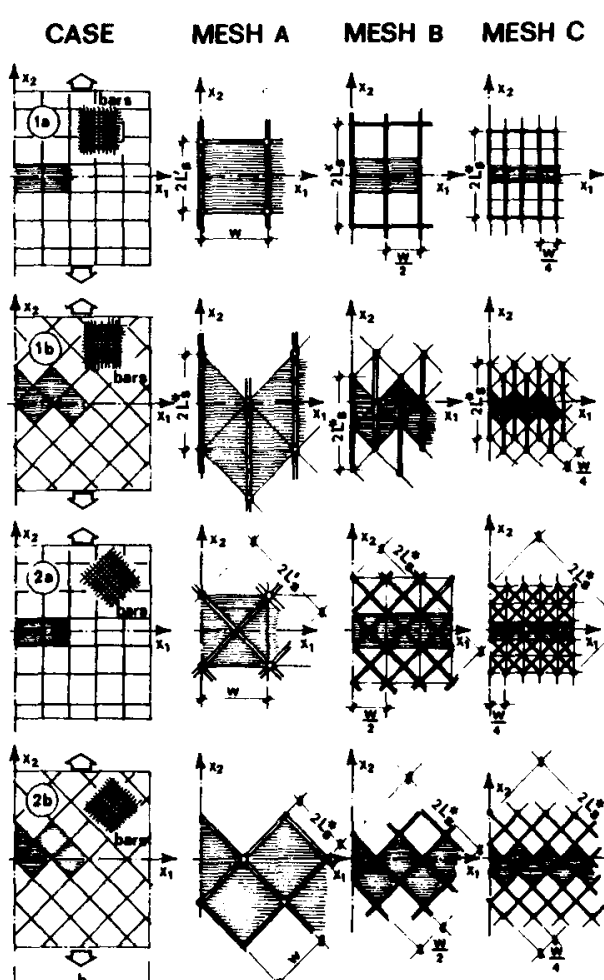

$\uparrow x_{2} \times$

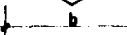

${ }^{7}$

$\frac{2}{2}$

龺

Fic 3-Finite Element Meshes and Reintorcing Grids Considered for CenterCracked Panels Analyzed in Examples and Refinement of the Element Meshes

manner as described in Ref. 2. The dimensions of the panel and the material properties are the same as in Ref. 2 . Three skew square meshes, with element sizes in the ratio 4:2:1, were considered (meshes $A, B$, and $C$ in Fig. 1). For the crudest mesh, the element size was $w=b / 7$ in which $b=$ half-width of the panel.

The results for the three skew meshes (case $1 b$ ), showing the value of the multiplier $\alpha$ of a unit load for which the crack band propagates, are presented in Fig. $4(b)$. The results from Ref. 2 for the parallel meshes are reproduced, for comparison, in Fig. 4(a). We see that the results for the three skew meshes are just as close to each other as they were found for the parallel meshes. This is true not only for plain concrete $(p=0)$ but also for reinforced concrete with reinforcement ratios $p=1.6 \%$ and $3.2 \%$.

Fig. 4(c) further shows a comparison of the result with the finest parallel and skew meshes. We also see that the mutual agreement is quite good here, for plain as well as reinforced panels.

It should be noted that the free bond slip length, defined in Ref. 2, was always taken equal to the distance between the nearest mesh nodes, and, for this reason, the cross section area of the bars crossing the crack
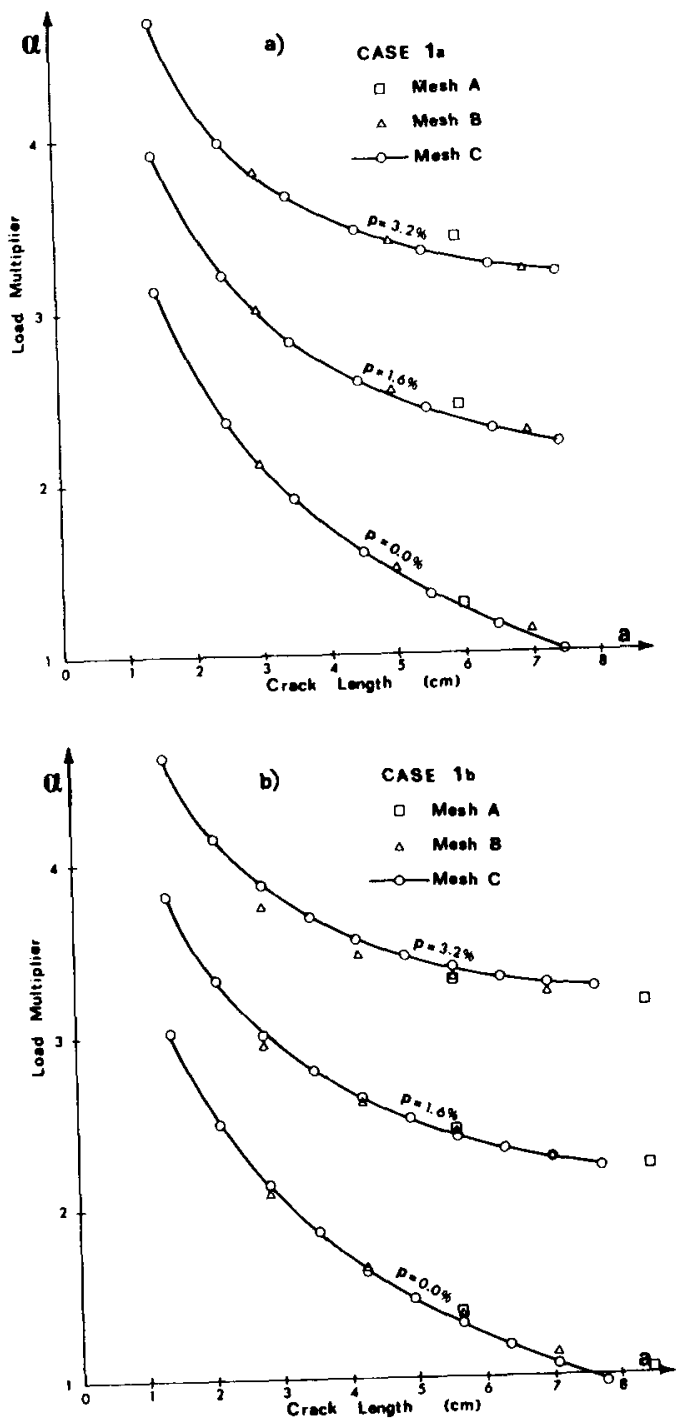

FIG. 4.- Numerical Resulte for the Center-Cracked Panels Defined In Fig. 3

band was adjusted according to Eq. 13 of Ref. 2. Because the distance across the crack band is different for the between the opposite nodes across the crack band is length, $L_{s}^{*}$, as cases of parallel mesh and skew mesh, the free bond slip length well as the cross section area of the bars within the free borallel mesh.

$A_{b}^{*}$, was not the same for the skew mesh as for the parallel mesh. We should also mention that, due to the need of limiting the number of elements and using an automatic mesh generation, the paneis analyzed with the three different meshes had slightly different dimensions. 

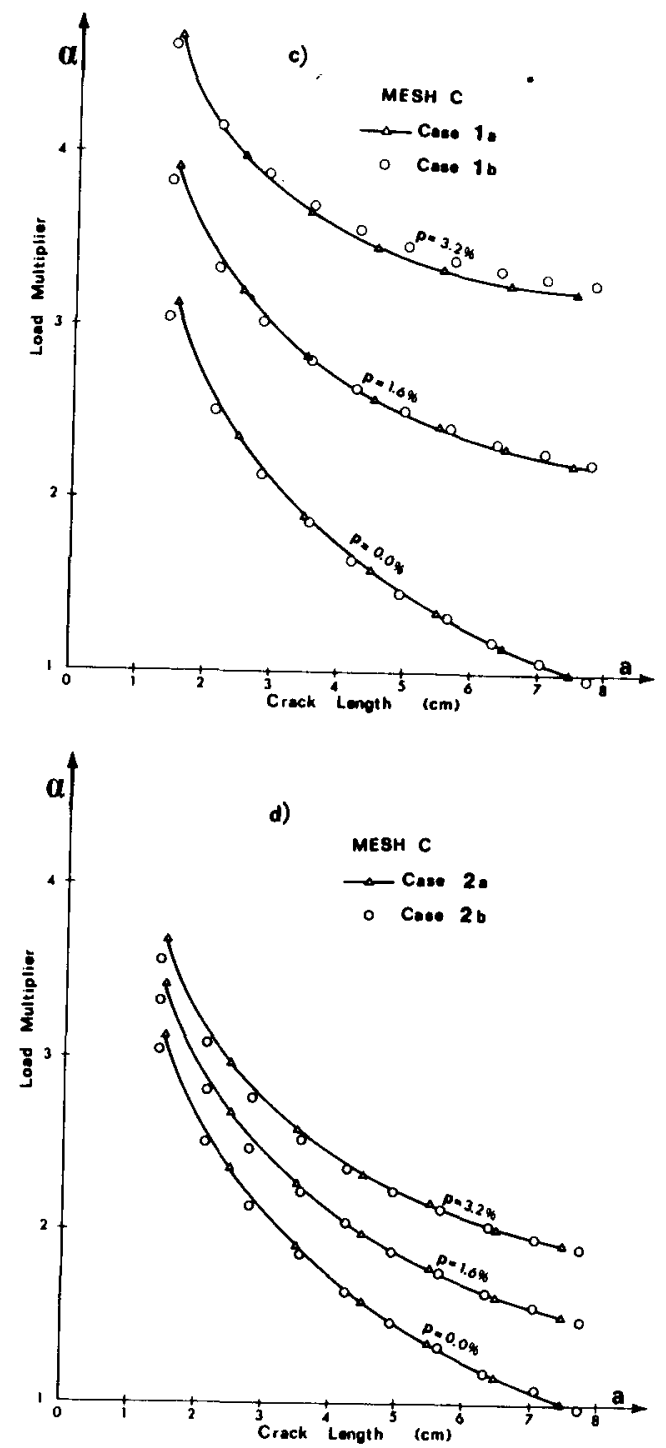

FIG. 4.-Continued

This is why the exact solutions for a vanishing element size (sharp crack), known of course only for plain concrete, are slightly different (Fig. 6).

Further results were obtained for the case of a mesh rotated to a 2:1 inclination (angle arctan 0.5), as shown in Fig. $5(a-c)$. In contrast to the previous cases, no symmetry with regard to the vertical axis could be exploited here to analyze only half of the panel. The numerical results for plain and reinforced panels are given in Fig. $5(d)$ for the three dif-
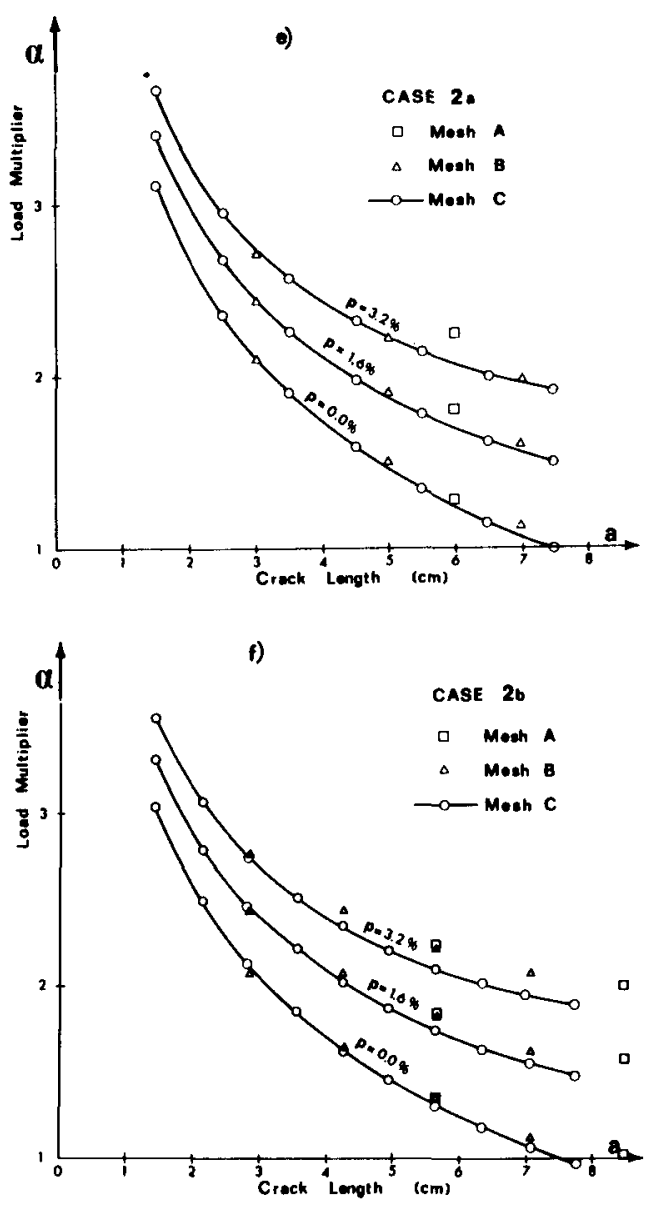

FIG. 4.-Continued

ferent meshes. Although the agreement between the results for these three meshes is not as good as in cases $1 a$ and $1 b$, the differences are acceptable. The larger jumps in Fig. $5(d)$ correspond to the case when the front cracked element moves from one inclined row to the next inclined row (Fig. 5(c)).

In addition to the comparison between two different mesh sizes ( $A$ and $B$ in Fig. $5(a$ and $b))$, Fig. $5(d)$ also shows a comparison with the results for the parallel mesh (case 1a) and the $45^{\circ}$-inclined mesh (case 1b). This agreement is also acceptable.

The foregoing results verify the objectivity and proper convergence of the method with regard to mesh rotation and a change of element size. For general applications, we also need to check the performance of the method when the reinforcing bars are neither orthogonal nor parallel to the crack direction. In this case (Fig. 2(e and $f)$ ), we need to consider 

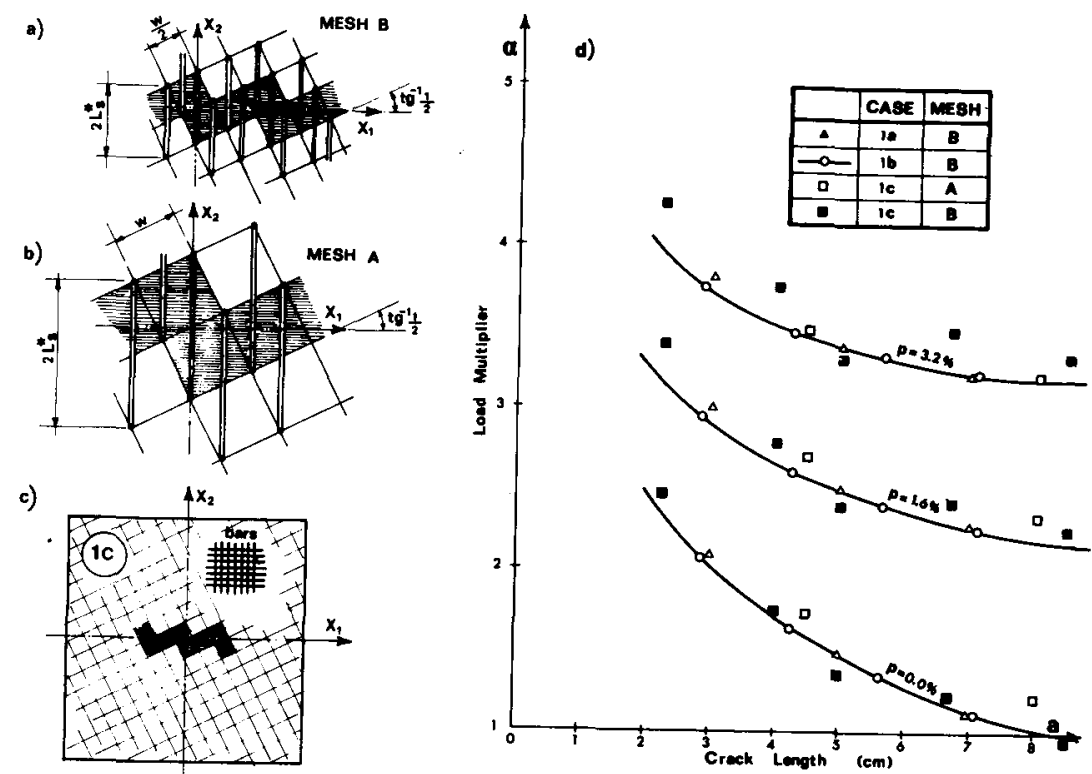

FIG. 5.-Center-Cracked Panel with 2:1 Mesh Inclination, Zig-Zag Crack Band, and Numerical Results

the questions of bond slip length and of the free bond slip length giving equal stiffness. Is the free bond slip length constant or does it depend on the angle between the bond and the cracks? It seems it does and should lie between the value $w$ used for orthogonal bars $(\alpha=0)$ and the value $w / \cos \alpha$ whose projection onto the normal to the crack preserves the length $L_{s}$. Numerical computations were made using the value $w /$ $\cos \alpha$, for which the projection of the free bond slip length onto the normal to the crack is kept constant (Fig. $2(f)$ ).

To check objectivity and convergence of the method for inclined reinforcing grids, the panels shown as cases $2 a$ and $2 b$ in Fig. 3 were considered. The comparisons between the results for various mesh sizes and various reinforcements are shown in Fig. 4(e) for the case of a parallel mesh and Fig. $4(f)$ for the case of a $45^{\circ}$ inclined mesh. The results for the finest parallel and inclined meshes are mutually compared in Fig. $4(d)$. In all cases, the agreement is acceptable, which confirms the objectivity and proper convergence of the method for the cases where the cracks are not normal to the reinforcing bars.

It is also interesting to compare the results for parallel and inclined reinforcements. We see that in the case of inclined reinforcement the results for various reinforcement percentages differ from each other much less, which seems to be caused by the fact that the inclined reinforcing grid is less stiff in the direction normal to the crack. This is partly due to Poisson's transverse contraction of the panel.

\section{Equivalent Strength in Presence of Reinforcement}

To allow easy application in the existing finite element programs for cracking of reinforced concrete, the equivalent strength criterion $(1,4)$ was previously formulated. According to this criterion, the strength limit for the principal stress normal to the crack direction in the element just ahead of the crack front must be adjusted according to the element size $w$. For plain concrete, the following criterion was derived;

$\sigma_{22}^{0} \leq f_{e q}=c \sqrt{\frac{E_{c}^{\prime} \mathscr{G}_{c r}}{w}}$

in which $\sigma_{22}^{0}=f_{\text {eq }}$ indicates that the crack extends, and $c$ is a coefficient characteristic of the given element type. For a square element composed of two constant strain triangles (Fig. $1(b)) c=0.921$ (Ref. 1), while for a square element consisting of four constant strain triangles (Fig. 1(c and d)) $c=0.826$ (Ref. 4).

Numerical studies have so far indicated that this criterion is satisfactory for the center-cracked panel without reinforcement. This is documented by the comparisons for the three different size meshes in Fig. 6 where the left diagram shows the results for the energy criterion and compares them to the exact solution available for the case of a vanishing element size, and the right diagram shows the comparisons with this exact solution for the equivalent strength criterion. It is seen that the deviations of the results for the equivalent strength criterion are only slightly more pronounced and still satisfactory.

For reinforced panels, it is found, however, that the deviations of the equivalent strength criterion predictions from those for the energy criterion increase with the reinforcement percentage and become quite substantial; see Fig. $6(d)$ in Ref. 2 , revealing an error of about $15 \%$ in the predicted load at propagation. This shows that an expression of the type of Eq. 3, even with a coefficient $c$ determined empirically so as to give the best fit of numerical results, is inadequate.

A more careful examination of the results for reinforced panels shows that the presence of reinforcement significantly decreases the crack opening near the crack front (see Fig. 6(a) of Ref. 2), compared to the opening which would be predicted by Eq. 18 of Ref. 1 from which the equivalent strength criterion was originally derived. This equation expresses the crack opening at a certain distance from the crack front on the basis of the first term of the asymptotic series expansion of the neartip displacement field of a sharp crack. One might at first think that the asymptotic series expansion could be applied also when the panel is reinforced, but this would be unjustified. The expansion is exact for the sharp crack $(r \rightarrow 0$, Fig. $7(b))$, and the presence of reinforcement can have no effect on the asymptotic expansion if the free bond slip length, $L_{s}^{\prime}$, is considered to be non-zero (which is a necessity). The reason: The leading term of the asymptotic expansion must be satisfactory within a small circle whose radius $r$ is sufficiently small (Fig. $7(b)$ ) compared to the free bond slip length because all forces coming from the reinforcement are then applied at points relatively remote from this circle.

We should also realize that, if we considered a smeared reinforcement with perfect bond (imagined as an adhering continuous layer, Fig. 7(a)), the asymptotic expansion for a sharp crack tip would of course not be applicable; but, in this case, we would get zero crack opening near the 
tip of a sharp crack because the reinforcing layer does not crack.

The inadequacy of Eq. 3 for reinforced concrete may be explained by the fact that the use of the first term of the series expansion is insufficient. Higher-order terms (see Eq. 23 of Ref. 1) are needed to describe the displacement field adequately. The second term of the asymptotic expansion corresponds to a uniform normal stress parallel to the crack, which has no effect on the crack opening and the transverse displacement. To improve the representation of displacements, we must consider, therefore, the third term, which is proportional to $r^{3 / 2} ; r=$ the
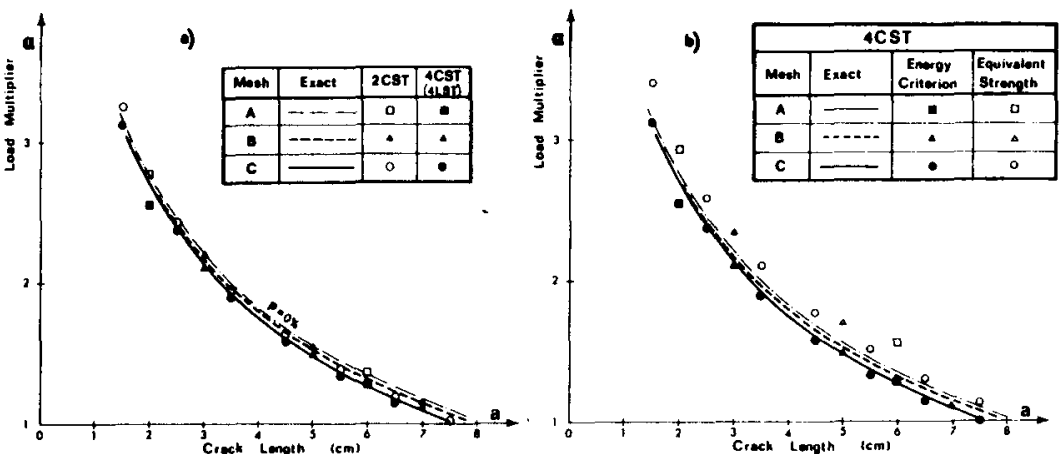

FIQ. 6.-Comparisons of Numerical Results for Center-Cracked Panel With Exact Solutions for Sharp Crack: (a) Energy Criterion; (b) Equivalent Strength Criterion

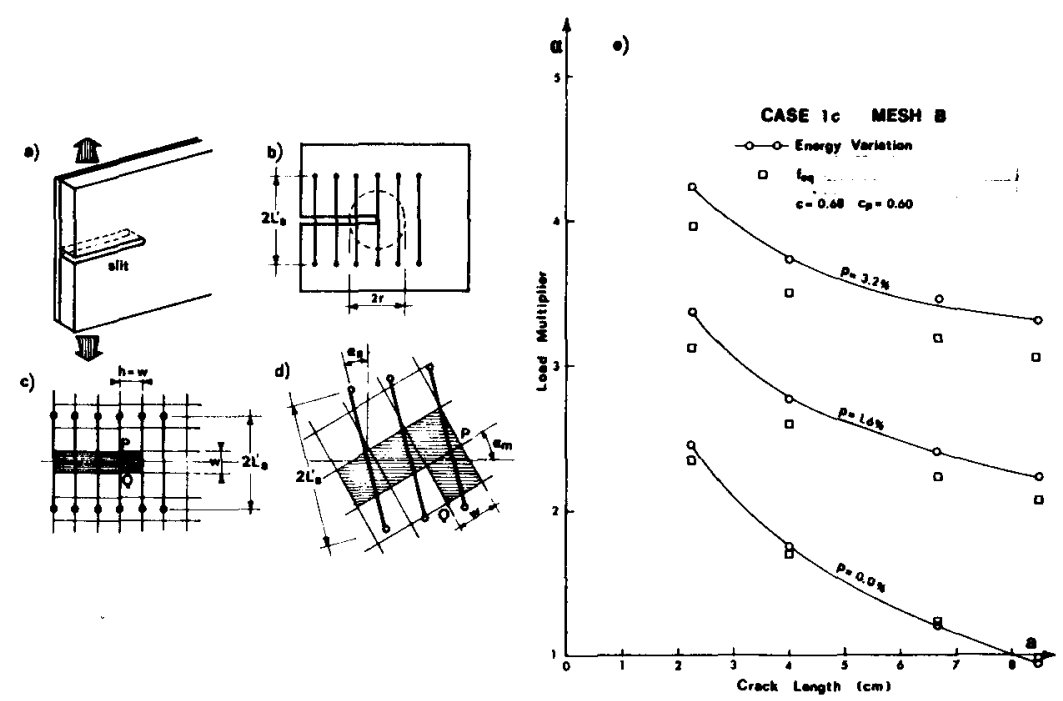

FIG. 7.-(a) Silt in Concrete Bonded to Uncracked Steel Layer; (b-d) Crack in Presence of Bond Slip; (e) Results of Equivalent Strength Criterion for the ZigZag Band from Fig. 5 distance from the tip of the equivalent sharp crack.

On the basis of the first three terms of the asymptotic expansion $(5,7)$ it can be shown that the relative displacement normal to the crack between two points $P$ and $Q$ of crack band front on opposite sides of the crack band (Fig. $7(c)$ ) is

$u_{\mathrm{P}}-u_{\mathrm{Q}}=8 \sqrt{\frac{\mathscr{G}_{c r}}{2 \pi E_{c}^{\prime}}} \sqrt{w}(1-\delta)$, with $\delta=c_{p} p \frac{E_{s}}{E_{c}^{\prime}} \frac{w}{L_{s}^{\prime} \cos \alpha_{s}}$

in which $\alpha_{s}=$ inclination of the reinforcement (Fig. $7(d)$ ) and $c_{p}=$ empirical dimensionless corrective factor. Note that, in the limit of a sharp crack $(w \rightarrow 0)$, Eq. 4 gives $u_{\mathrm{P}}-u_{\mathrm{Q}}=0$, which must be so since points $\mathrm{P}$ and $\mathrm{Q}$ become identical to the crack tip when $w=0$.

The form of the corrective term, $\delta$, has the following effects: It reduces the opening displacement in proportion to the stiffness of the bars relative to concrete (i.e., $p E_{s} / E_{c}^{\prime}$ ); it reduces the crack opening displacement in proportion to the distance of the points from the crack tip, assumed to be fixed relative to $w$ (Fig. 7(c and $d)$ ); and it reduces the crack opening in inverse proportion to the free slip length projection onto the normal of cracks (Fig. $7(c$ and $d)$ ). The corrective term satisfies the limiting conditions that for $p \rightarrow 0$, or $E_{s} \rightarrow 0$, or $w \rightarrow 0, L_{s}^{\prime} \rightarrow \infty$, or $\alpha_{s} \rightarrow 90^{\circ}$, the formula for plain concrete must be recovered.

To obtain an expression for the equivalent strength $f_{\text {eq }}$, we may now use the approximate relation $\xi_{c r} \simeq c_{f} f_{e q}\left(u_{\mathrm{p}}-u_{\mathrm{Q}}\right)$ in which $c_{f}$ is an empirical factor close to 1.0. Substituting Eq. 4 here and using the approximation $1 /(1-\delta) \simeq 1+\delta$ (for $\delta<<1$ ), we obtain for the equivalent strength the following formula:

$f_{e q}=c\left(\alpha_{m}\right) \sqrt{\frac{E_{c}^{\prime} \xi_{c r}}{w}}\left[1+c_{p}\left(\alpha_{s}\right) p n \frac{w}{L_{s}^{\prime} \cos \alpha_{s}}\right]$

in which $n=E_{s} / E_{c}^{\prime} ; c$ and $c_{p}$ are coefficients to be determined empirically so as to achieve the best fit of numerical results. We indicate in the formula that these coefficients must be functions of the inclination angle of the mesh, $\alpha_{m}$ and the inclination angle of the reinforcing grid, $\alpha_{s}$ (Fig. $7(d))$. Note that the expression $1+\delta$ is preferable over $1 /(1-\delta)$ since it cannot become negative for large $\delta$, while $1 /(1-\delta)$ can.

To determine the values of $c\left(\alpha_{m}\right)$ and $c\left(\alpha_{s}\right)$ for $\alpha_{m}=0^{\circ}, 45^{\circ}$ and $\alpha_{s}=$ $0^{\circ}, 45^{\circ}$, the results for the center-cracked panel obtained with the energy criterion were fitted. Optimum fits have been obtained using the Marquardt-Levenberg algorithm, which minimizes the sum of squares of the

TABLE 1.-Coefficlents for Reinforcement Effect on Equivalent Strength

\begin{tabular}{l|c|c|c|c|c}
\hline \hline \multirow{2}{*}{\begin{tabular}{c} 
Coefficient \\
\multicolumn{1}{c|}{$(1)$}
\end{tabular}} & $\begin{array}{c}|c| \\
\text { Case 1a } \\
(2)\end{array}$ & $\begin{array}{c}\text { Case 1b } \\
(3)\end{array}$ & $\begin{array}{c}\text { Case 2a } \\
(4)\end{array}$ & $\begin{array}{c}\text { Case 2b } \\
(5)\end{array}$ & $\begin{array}{c}\text { Assumed } \\
(6)\end{array}$ \\
\hline$c\left(0^{\circ}\right)$ & 0.75 & - & 0.74 & - & 0.74 \\
$c\left(45^{\circ}\right)$ & - & 0.62 & - & 0.61 & 0.62 \\
$c_{p}\left(0^{\circ}\right)$ & 0.61 & 0.60 & - & - & 0.60 \\
$c_{p}\left(45^{\circ}\right)$ & - & - & 0.32 & 0.45 & 0.40 \\
\hline \hline
\end{tabular}


deviations from the results of the energy criterion. This was done for cases $1 a, 1 b, 2 a$, and $2 b$, and the results are listed in Table 1 in the first four columns.

We see that the results for normal and skew reinforcement are quite close to each other, which supports the assumed dependence of $c$ on $\alpha_{n}$ and $c_{p}$ on $\alpha_{s}$. The values of the empirical coefficients which were determined on the basis of these results are indicated in the last column of Table 1. The results for the center-cracked panel, plain and with normal or skew reinforcement, are plotted in Figs. 8 and 9. We see in these figures a satisfactory agreement with the energy criterion.

Numerical results have also been obtained for case $1 \mathrm{c}\left(\alpha_{m}=\arctan 0.5\right.$ $\alpha_{s}=0^{\circ}$ ) (Fig. 7(e)). In these calculations in which linear interpolation between the value $c(0)$ and $c\left(45^{\circ}\right)$ has been assumed, the agreement is not too close, but still acceptable for practical purposes. We should also realize, however, that for this case the results previously obtained for the energy criterion (Fig. 5(d)) were somewhat higher than those obtained here for cases $1 a$ and $1 b$, which offsets the error apparent from Table 1.

On the basis of the numerical results for one type of fracture specimen, namely the center-cracked panel, we of course cannot assert that Eq. 5 should be generally valid for arbitrary geometries of the specimen or structure. We may expect it, however, based on the fact that Eq. 5 has its theoretical foundation in Eq. 4.

\section{Various Types of Chacked Specimens}

It is known from fracture mechanics that various types of fracture test specimens, which simulate various characteristic situations in structures, can often give rather different results. Agreement with all of them, required for any general method, is difficult to achieve. All previous numerical work on the blunt smeared crack band approach has been confined to the center-cracked panel $(1,2,4)$. Therefore, the method has now been applied to another specimen, the three-point bent specimen (Fig. 10(a)). In this specimen, there is a higher stress gradient throughout the ligament in front of the crack band than in the center-cracked tensile specimen, and the stresses change sign from tensile to compressive, which does not happen in the center-cracked specimen. This type of specimen has often been used for fracture tests of concrete.

Here, we analyze the specimen used by Walsh (7), the largest one of his first series, the dimensions of which were (Fig. 10(a)) $(L=40$ in. $(1,016 \mathrm{~mm}) ; b=3$ in. $(76 \mathrm{~mm}) ; d=15$ in. $(381 \mathrm{~mm}) ; a=5$ in. $(127 \mathrm{~mm})$; the modulus of rupture was 786 psi $(5,416 \mathrm{kPa})$, the compressive strength was $3,350 \mathrm{psi}(23,082 \mathrm{kPa})$, and the failure load was measured as $P=2,095 \mathrm{lb}(9,323 \mathrm{~N})$. The expression used by Walsh for the stress intensity factor of a sharp crack in the specimen is $K_{l}=3.4 \mathrm{~Pb}^{-1}(d-a)^{-1 /}$

${ }^{2}$. This is an approximate elasticity solution, which is slightly lower (as it should be) than the one calculated from the formulas given by Knott (5) and Tada (7) for the case of a somewhat more slender beam having $L / d=4$, for which they give $K_{I}=3.6 \mathrm{~Pb}^{-1}(d-a)^{-1 / 2}$. The value used by Walsh has been adopted for comparing the finite element results as the element size tends to zero. The energy release rate $\mathscr{G}$ can be ex-
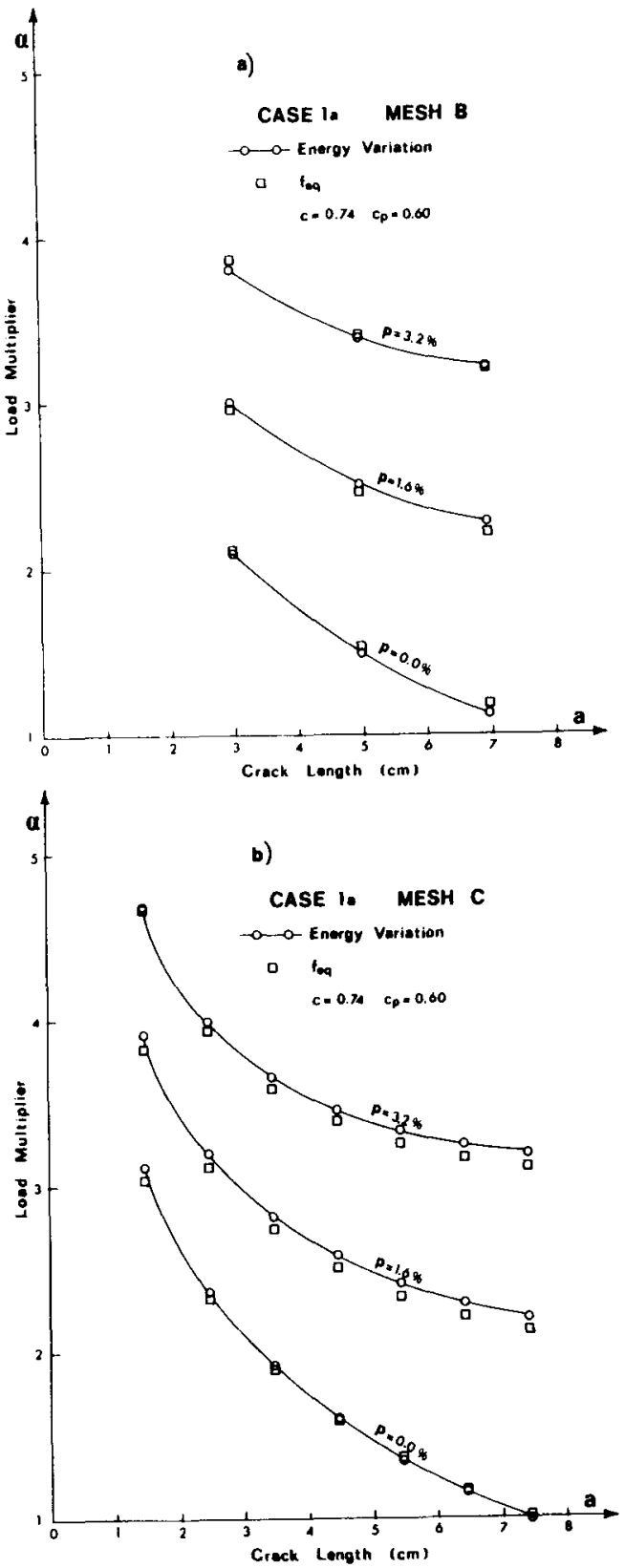

FIG. 8.-Results of Equivalent Strength Criterion for Cases 1a and 1b from Fig. 3 

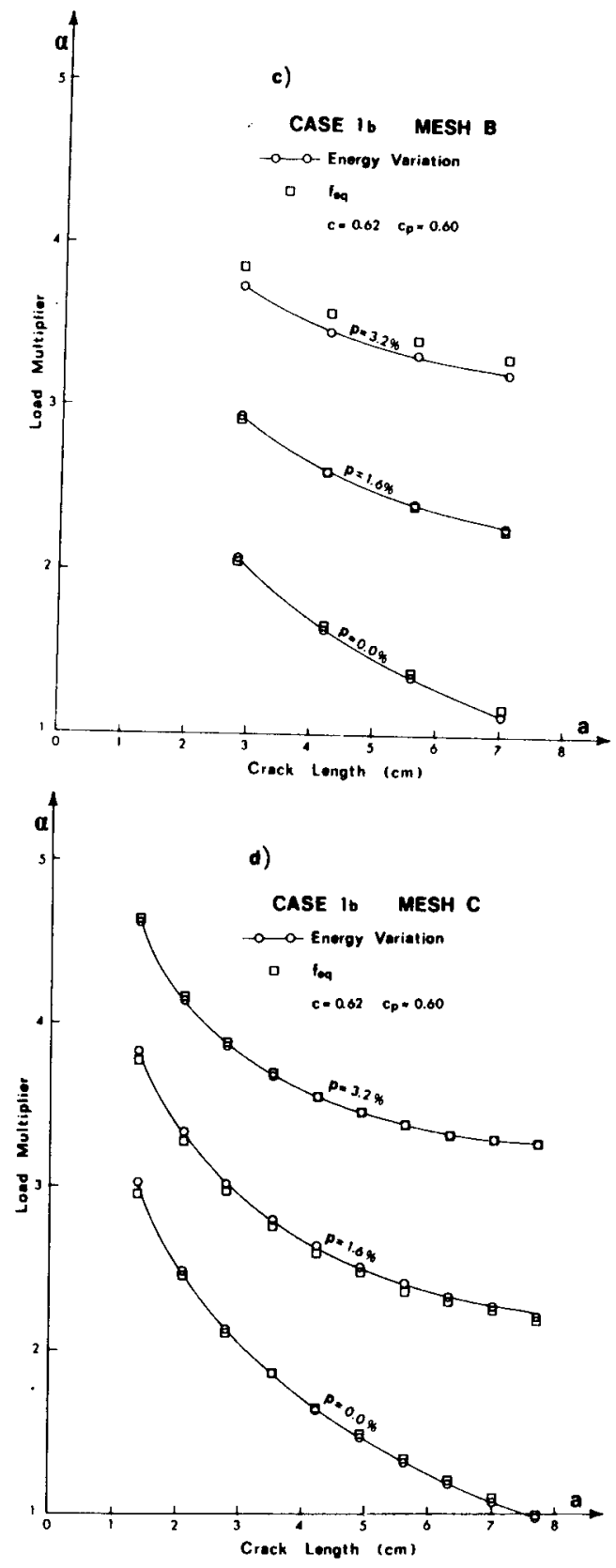

FIG. 8.-Continued
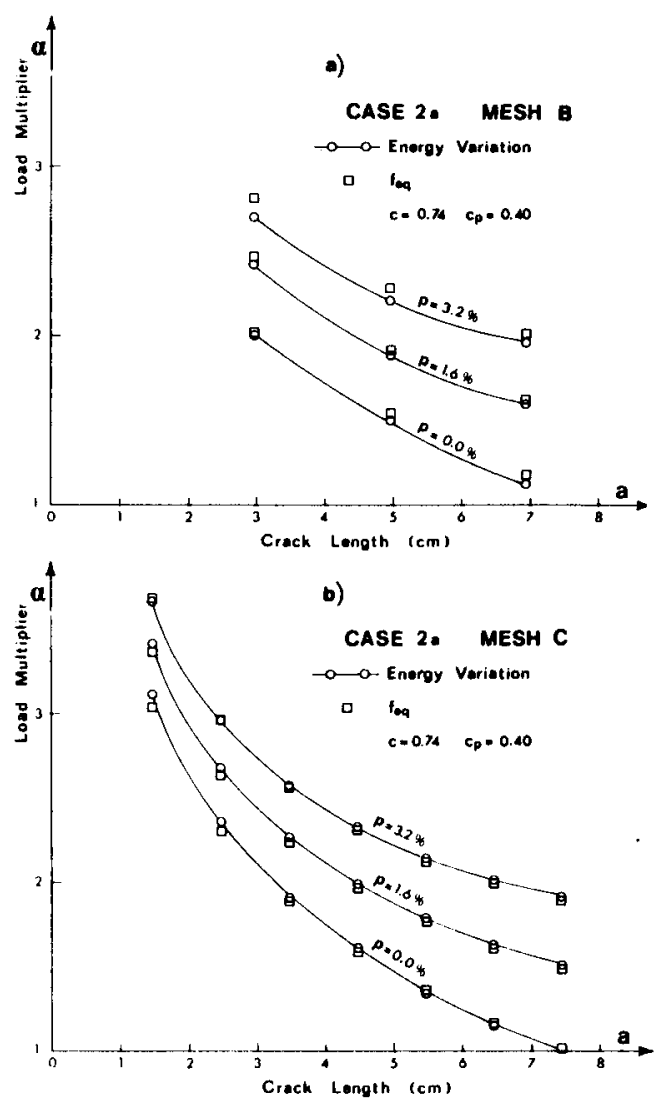

FIG. 9.-Results for Equivalent Strength Criterion for Cases 2a and 2b from Fig. 3

pressed, as known from fracture mechanics (4), as $\mathscr{G}=K_{I}^{2} / E$. As the applied load, the experimentally measured failure load has been considered and the critical value of the stress intensity factor reported by Walsh (in his Eq. 2.5) has been adopted.

The results for the load multiplier which causes further crack extension are summarized in Table 2 for the three meshes of sizes 4:2:1. For an exact agreement with Walsh, the value of load multiplier $\alpha$ has to be 1.0, and so deviations from 1.0 are an indication of the relative error. We see from Table 2 that the results for the energy variation criterion are, for this type of specimen, again in excellent agreement. This indicates that the energy variation method is of general applicability. (It should be mentioned that in order to simplify computations the meshes in Fig. 10(b-d) were not uniform over the entire length of the beam; the meshes are finer in the region surrounding the crack band front.)

Looking at the results for the equivalent strength criterion in Table 2, however, we see more serious discrepancies, both with regard to the elasticity solution used by Walsh as well as among the different meshes. 

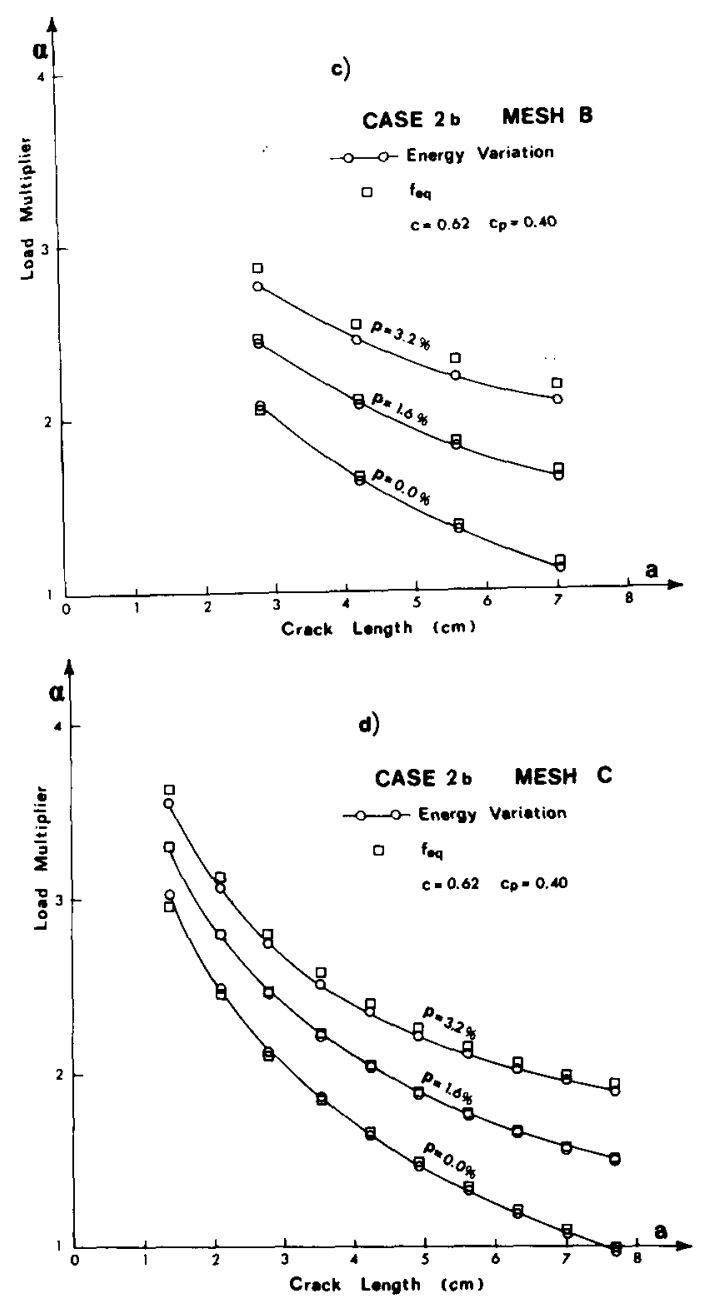

FIG. 9.-Continued

Although we see a strong improvement of the results as the mesh is refined, the results are not very good even for the finest mesh (15\% error). This indicates that, for this type of specimen, the equivalent strength criterion gives accurate results only with very fine meshes. For the meshes suitable for practical use (Fig. 10), one has to accept an error which is more serious, albeit not unacceptable for many applications. It is worth noting, however, that the results for the equivalent strength appear to converge to the exact solution (i.e., 1.0).

The need for refined meshes when the equivalent strength criterion is applied to bent specimens is apparently due to the fact that the gradient of normal stress throughout the ligament ahead of the crack front is high and the stresses change from tensile to compressive, while in

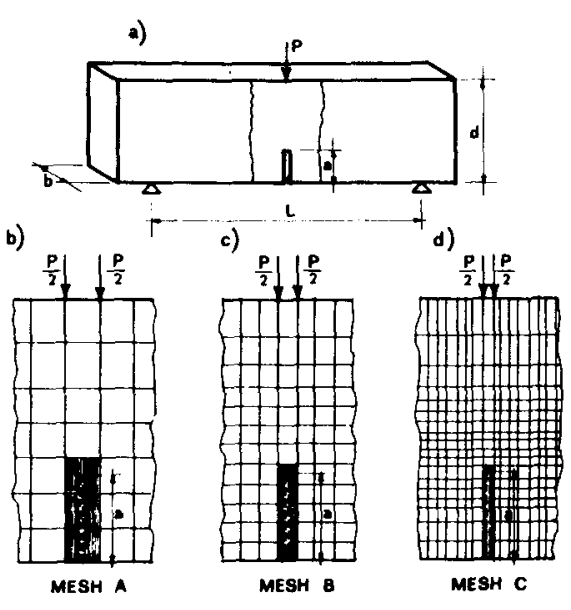

FIG. 10.-Three-Point Bent Specimen and Finite Element Meshes Used in Examples

the center-cracked specimen the gradient is less and the normal stress ahead of the crack front tends to a constant value.

It should be also noted that if the value of the tensile strength is considered as 2/3 of the modulus of rupture, then the use of the tensile strength criterion gives almost correct results for the element size shown in Fig. 10(c). As already pointed out in Ref. 1, this may be one of the reasons good results are often obtained by finite element analysis of reinforced concrete beams based on the tensile strength criterion.

\section{Various Element Types and Other Aspects}

Various Types of Elements.-For practical application, the question of optimum element type is of interest. With regard to the question of using higher-order elements, we should note that cracking represents a discontinuous change with a sharp jump in the strain values. For the same number of degrees of freedom, the higher-order elements are of a larger size; since a jump in strain can be represented only at the element boundary, the use of higher-order elements enforces a poorer, coarser representation of fracture discontinuities, i.e., a poorer resolution. It, thus, seems inadvisable to use higher-order finite elements, with higher-order polynomial distribution functions, for the modeling of fracture.

TABLE 2.-Load Multipller a Computed from Varlous Cracking Criteria for ThreePoint Bent Specimen

\begin{tabular}{c|c|c|c}
\hline $\begin{array}{c}\text { Mesh } \\
\text { (1) }\end{array}$ & $\begin{array}{c}\text { Energy } \\
\text { variation } \\
(2)\end{array}$ & $\begin{array}{c}\text { Equivalent } \\
\text { strength } \\
(3)\end{array}$ & $\begin{array}{c}\text { Constant } \\
\text { strength } \\
(4)\end{array}$ \\
\hline A & 0.977 & 1.399 & 1.672 \\
B & 0.981 & 1.234 & 1.099 \\
C & 0.988 & 1.147 & 0.722 \\
\hline
\end{tabular}


The previous studies focused on the simplest constant-strain elements, two of which were combined in a square element (1), and on quadrilateral elements composed of four linear strain triangles in which the midside nodes on the square were constrained by a linear displacement distribution along the side of the square (Fig. $1(b$ and $d)$ ). It was also shown (3) that, for an equally fine mesh, the use of linear, instead of constant, strain triangles makes the system more flexible, giving rise to slightly smaller values of the load multiplier $\alpha$. The same trend is recognizable in passing from a coarser to a finer mesh with constant strain triangles (see Fig. 3(b) of Ref. 1). This observation is not unexpected since both the mesh refinement and the use of higher-order elements leads to a greater number of degrees of freedom. From the calculations made, it appears, however, that the use of linear strain triangles did not appear to improve, in general, on the results obtained with the constant strain triangles.

So the use of constant strain triangles, which are much simpler, seems to be acceptable. The square elements consisting of two triangles exhibit however, a certain directional bias due to the slope of the diagonal (Fig. $1(b))$. Therefore, the quadrilateral element made of four constant strain triangles (4CST, Fig. 1(c)) was tried in the calculation. These results are shown in Fig. 6(a), together with the exact solution for the sharp crack. (Note that the panels analyzed with different meshes have slightly different dimensions and consequently slightly different exact solutions.) As expected, the results for the four-triangle quadrilaterals are lower than those for the two-triangle quadrilaterals. Moreover, they are almost undistinguishable from the results for quadrilaterals consisting of four linear strain triangles; for this reason, they are marked with the same symbol in the figure. The reason may be that the linear strain triangles have been constrained to constant strain on the boundary of the square. Fig. 6(a) demonstrates again the objectivity of the method with regard to the element size for both types of finite elements used.

As for the equivalent strength criterion, the quadrilaterals consisting of four linear strain triangles give results higher than the correct ones obtained with the energy criterion. This is due to the assumptions made in deriving the equivalent strength criterion, as already examined in Ref. 3. What should be noted, however, is the fact that the equivalent strength criterion in this example gives fairly good results even with a relatively coarse mesh.

Bond-Slip Representation.-Another practical modeling question arises with regard to the representation of bond slip. As shown in previous work (2), a simple model is that of free bond slip length, which gives the correct overall stiffness for the connection between the opposite sides of the crack band. The free bond slip length, $L_{s}^{\prime}$ (Fig. 11(b)), usually does not coincide with the distance between two nodes in the mesh Interpolation for the displacement at the end of the free bond slip length is then necessary, and a special element was for this purpose described in Appendix I of Ref. 2. When this procedure is used, it is inconvenient to introduce the free bond slip length as the crack band extends through a certain element. For this reason, the free bond slip was assumed in the previous work (2) to exist in advance in all the elements through which the crack is expected to pass (Fig. 11(c)). It was checked of course

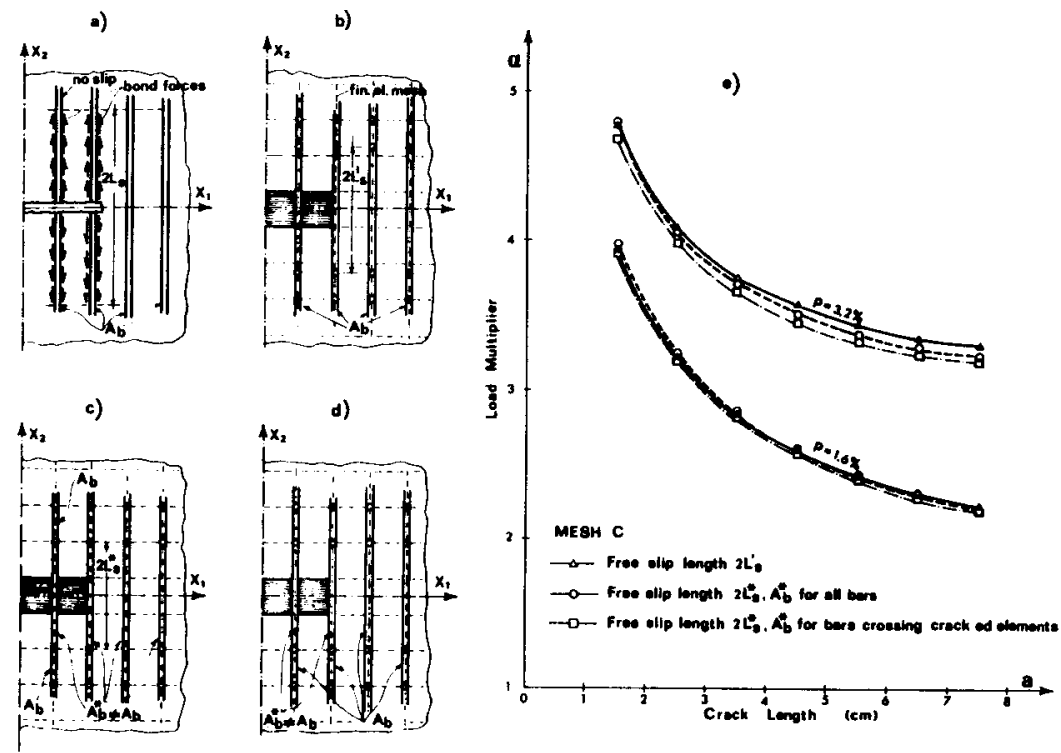

FIG. 11. (a) Actual Bond SIlp of Bars at Crack Crossing; $(b-d)$ Mesh Refinements Used in Examples; (e) Comparison of Numerical Results

that this makes little difference from the more correct assumption, namely, that there is no bond slip in the elements which have not cracked yet.

A more realistic representation, in which the bond slip is introduced only after the element cracks, has been proposed in Ref. 2 but was not considered in the numerical results reported there. In this method, the cross section of the bars crossing the crack band, $A_{b}$ is adjusted to a certain value, $A_{b}^{*}$, (2) for which the free bond slip length coincides with the distance between certain two nodes and the overall stiffness of the connection between the opposite sides of the crack band remains the same. With this representation, it becomes simple to introduce the free bond slip not in advance but during the computation, as the crack band passes through the given element (Fig. 11(d)). Numerical results obtained with this representation of the bond slip are presented in Fig. 11(e). Now, for the same panel, as specified in Fig. 1(a), and for mesh $C$, these results confirm that the previously used approximation with free bond slip considered in advance in all elements expected to crack introduces only a small, hardly detectable, error.

It should be realized that at the time the bond slip is introduced together with a change in the area of the bars, a part of the energy change of the entire structure is due to the change in stiffness of the bar within the bond slip length. Of course only the energy due to cracking alone must be included in Eqs. 1 and 2. For the same panel, the computations then yield the results plotted in Fig. 11(e).

Work Dissipated By Bond Slip.-Not all of the energy supplied to the cracking element (volume $\Delta V$ ) from the rest of the structure and 
from the unloading of concrete between the cracks is available for producing new crack surfaces. Part of this energy is consumed by the bond slip of reinforcing bars during cracking within volume $\Delta V$, and only the rest of the energy is available for producing the crack surfaces. Therefore, in a more rigorous formulation we should subtract the energy consumed by the bond slip, in which the previous expression for the energy release (2) rate may be corrected as

$\mathscr{G}=\frac{1}{\Delta a}\left[\Delta W_{\Delta V}+\Delta L-\iint_{0}^{\delta_{b}} U_{b}^{\prime}\left(\delta_{b}\right) d \delta_{b} d s\right]$

Here, $\delta_{b}=$ the relative tangential displacement between the bars and the concrete; $U_{b}^{\prime}=$ the ultimate bond force per unit length of the bar (force during the slip); and $s=$ the length of the bar segment within the fracture process zone (and not within volume $\Delta V$ since the energy consumed by bond slip would then depend on the chosen element size and would, thus, spoil the objectivity and proper convergence of the fracture criterion).

At this point, we can see, however, various uncertainties in evaluating the energy consumed by bond slip. First, if we imagine the cracks to be perfectly continuously distributed, it is possible that no debonding and bond slip take place within the fracture process zone; they would occur only behind the crack front where the distributed cracks coalesce into one large visible crack. Second, since the free slip length is normally longer than the finite element width, our model does not involve any bond forces between the bar and the concrete within the fracture process zone; so we have to evaluate the bond slip term in Eq. 6 independently of our model.

Furthermore, with regard to our free bond slip concept, we must keep in mind that, properly, an additional energy correction should be made due to the fact that the energy balance in the entire structure is affected by replacing the actual bond slip length $L_{s}$ and the actual bar cross section $A_{b}$ with the free bond slip length $L_{s}^{*}$ and the modified cross section area of the bar $A_{b}^{*}$. Intuitively, it seems that the corrections just mentioned might not be too important, but this should be checked more carefully in further research.

Cracking of Shear Panel.-It is interesting to examine objectivity of the analysis for the shear failure of the panel in Fig. 12, which is reinforced only by flexural steel concentrated near the bottom of the panel, and is loaded by a vertical force at midspan. Contrary to the examples presented so far, the crack band path is not known in advance but is to be found. We assume the crack band to spread into that element (adjacent to the crack front element) in which the principal tensile stress is the largest and equals or exceeds the actual tensile strength $f_{t}^{\prime}$ or the equivalent strength $f_{e q}$. The displacement at the loading point is introduced in small increments. At each load step, Newton-Raphson iterative procedure is used to redistribute the unbalanced modal forces due to cracking until a stable crack band configuration is reached. Linear elastic behavior is assumed for concrete in compression. The detailed data used for the analysis are given in the discussion and closure of Ref. 2 .

The finite element analysis was carried out for three different meshes

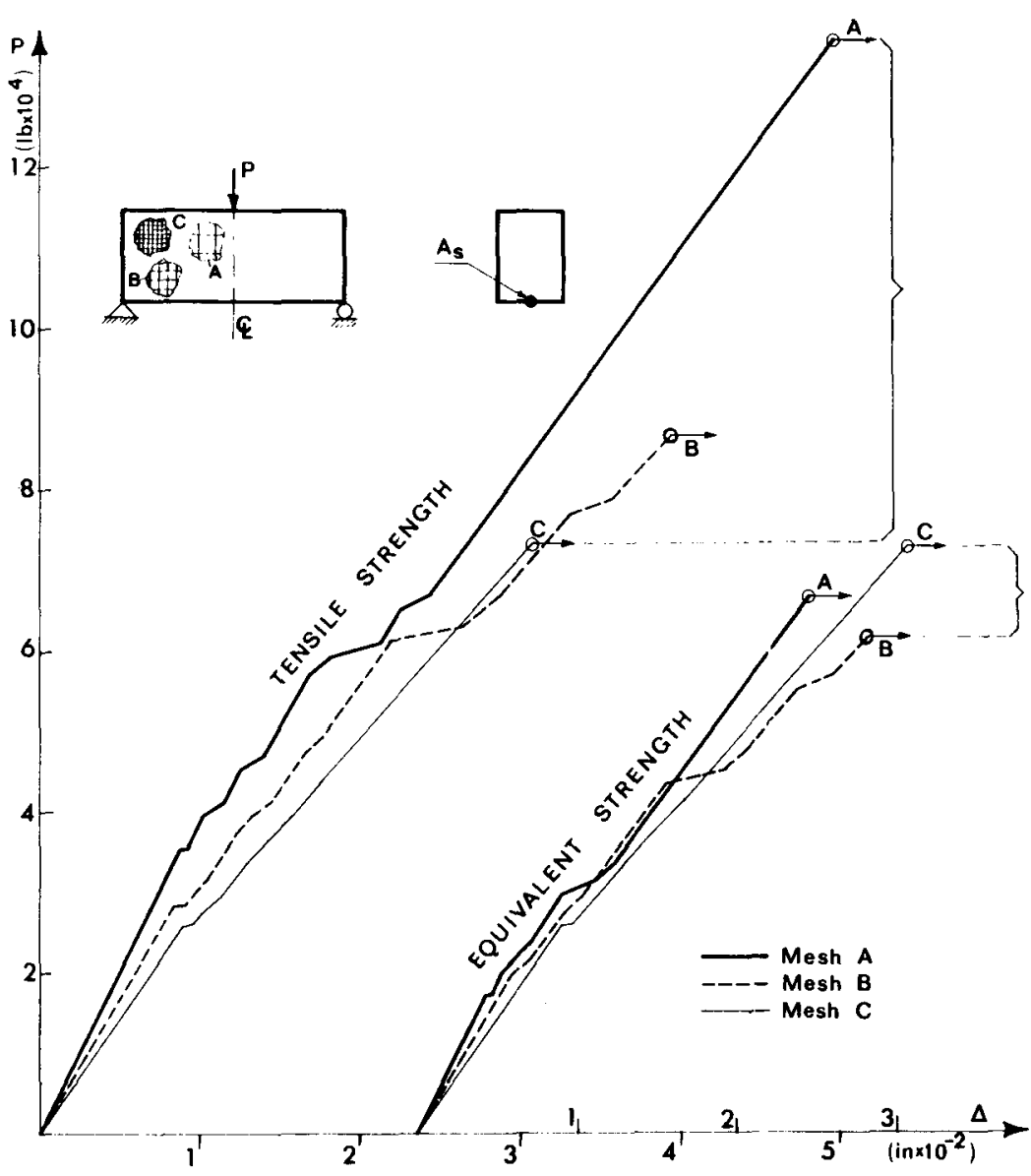

FIG. 12.-Load Deflection Curves of Shear Panel for Different Meshes

(A, B, and C in Fig. 11), the mesh sizes of which are in the ratio 4:2:1. The load-deflection curves obtained for these three meshes are plotted in Fig. 12. Even though this problem is less sensitive to the value of tensile strength than are the problems considered before, we see that the deflections curves are more consistent for the equivalent strength criterion. More sensitive is the value of the load at which the crack zone reaches a certain fixed distance from the top. We fix this distance as onehalf the size of the element in the crudest mesh (A), which then equals a distance of one element for mesh B, and of two elements for mesh C. The loads for which the crack zone reaches this distance from the top are indicated in Fig. 12 by horizontal arrows, and we see that for the equivalent strength criterion they differ from each other much less than they do for the fixed tensile strength criterion. Furthermore, it is found (see closure of Ref. 2) that the crack patterns for meshes A, B, and C are rather different and, in particular, the crack zone for the finest mesh 
is not diffuse but localizes into narrow, separate crack bands of single element width at the front. This behavior is obtained, however, only when the loading steps are taken to be so small that no more than one element cracks during the first iteration of each loading step.

\section{Summary and Conclusions}

Propagation of a blunt smeared crack band through a finite element mesh is analyzed for plain as well as reinforced concrete structures. The previous work in which an energy criterion for the crack band advance was formulated is extended by examining the effects of mesh rotation, reinforcement, bond slip, and the question of equivalent strength. The principal conclusions are the following:

1. The objectivity and proper convergence of the energy criterion, which was previously demonstrated with regard to variations in the mesh size for parallel meshes and orthogonal reinforcing bars, is numerically verified here for changes in the inclination of the mesh or the reinforcing bars with regard to the crack direction.

2. Modeling of the bond slip with the help of the free bond slip length leads to objective and properly convergent results for the cases of arbitrarily inclined mesh or inclined reinforcing bars.

3. In presence of reinforcement, the previously published formula for the equivalent strength may be improved by introducing a correction which corresponds to the third term of the asymptotic expansion of the displacement near a sharp crack tip. The corrective term depends on the reinforcement ratio, the elastic modulus of steel, the bond slip length, the element width, and the inclination of the bars.

4. The energy criterion gives consistent results for various types of fracture specimens, in particular the center-cracked specimen and the three-point bent specimen. This criterion, thus, seems to be of general applicability.

5. The equivalent strength calibrated for the center-cracked tensile specimen leads (even with a fine mesh) to a substantial error (about $15 \%$ ) when applied to the three-point bent specimen. So this method is inferior in accuracy to the energy criterion, although convergence to the correct solution seems to be verified. Due to its simplicity, the equivalent strength concept is, however, so attractive that its use might be justified in practical applications when fine meshes are used. Errors of approx $15 \%$ should then be expected. This is still much better than the errors of approx $100 \%$ found for the usual constant strength criterion.

6. In formulating the energy criterion, the work consumed by the slip of bars within concrete in the fracture process zone should be subtracted from the energy made available to form the cracks. This correction seems, however, to be relatively unimportant.

\section{ACKNOWLEDGMENT}

Partial financial support by National Science Foundation under Grant CME8009050 to Northwestern University is gratefully acknowledged.

\section{ApPendix.-References} 1. Bažant, Z. P., and Cedolin, L., "Blunt Crack Band Propagation in Finite Ele-
ment Analysis," Journal of the Engineering Mechanics Division, ASCE, Vol. 105, No. EM2, Apr., 1979, pp. 297-315.

2. Bažant, Z. P., and Cedolin, L., "Fracture Mechanics of Reinforced Concrete," Journal of the Engineering Mechanics Division, ASCE, Vol. 106, EM6, Proc. Paper 15917, Dec., 1980, pp. 1287-1306; discussion by David Darwin and Robert Dodds, Vol. 108, No. EM2, Apr., 1982, pp. 464-466; closure, Vol. 108, No. EM2, Apr., 1982, pp. 466-471.

3. Bažant, Z. P., and Oh, B. H., "Concrete Fracture Via Stress-Strain Relations, Report No. 81-10/665, Center for Concrete and Geomaterials, Technological Institute, Northwestern University, Evanston, Ill., Oct., 1981.

4. Cedolin, L., and Bažant, Z. P., "Effect of Finite Element Choice in Blunt Crack Band Analysis," Computer Methods in Applied Mechanics and Engineering, Vol. 24, 1980, pp. 305-316.

5. Knott, J. F., Fundamentals of Fracture Mechanics, Butterworths, London, England, 1973, p. 132.

6. Rice, J. R., "Mathematical Analysis in the Mechanics of Fracture," Fracture, an Advanced Treatise, H. Liebowitz, ed., Vol. 2, Academic Press, New York, N.Y., 1968, pp. 191-250.

7. Tada, H., The Stress Analysis of Cracks Handbook, Del Research Corp., Hellertown, Pa., 1973, p. 2.16

8. Walsh, P. F., "Fracture of Plain Concrete," The Indian Concrete Journal, Vol. 46, Nov., 1972, pp. 469-471. 